\title{
Types of social media use and digital stress in early adolescence
}

\author{
Lizzy Winstone $^{1}$, Becky Mars ${ }^{1,2}$, Claire M.A. Haworth ${ }^{2,3,4}$, and Judi Kidger ${ }^{1}$ \\ ${ }^{1}$ Population Health Sciences, Bristol Medical School, University of Bristol, UK \\ ${ }^{2}$ NIHR Biomedical Research Centre at the University Hospitals Bristol NHS Foundation \\ Trust \\ ${ }^{3}$ School of Psychological Science, University of Bristol, UK \\ ${ }^{4}$ The Alan Turing Institute, British Library, London, UK
}

Correspondence to: Lizzy Winstone, Population Health Sciences, Bristol Medical School, University of Bristol, BS8 2BN, UK; $\underline{\text { lizzy.winstone@bristol.ac.uk }}$

This preprint was published in Journal of Early Adolescence on 25 ${ }^{\text {th }}$ May 2022; https://doi.org/10.1177/02724316221105560

Lizzy Winstone is Senior Research Associate at the University of Bristol with an interest in young people's mental health. Lizzy's mixed methods research explores different types of social media use in early adolescence, and relationships with anxiety, depression, self-harm, well-being, and social connectedness. http://orcid.org/0000-0002-5547-6087

Becky Mars is a Research Fellow in Epidemiology at the University of Bristol. Becky's research focuses on the epidemiology and course of self-harm and suicidal behaviours, 
including transitions between suicidal thoughts/non-suicidal self-harm and suicide attempts. http://orcid.org/0000-0002-8132-6920

Claire Haworth is a Professor in Behavioural Genetics at the University of Bristol. Claire is interested in what factors influence our mental health and wellbeing across the life course. Claire is also interested in the use of information from social media platforms, and what benefits and challenges social media use has for mental health and well-being. http://orcid.org/0000-0002-8608-289X

Judi Kidger is a Senior Lecturer in Public Health at the University of Bristol. Her research expertise lies in young people's mental health and self-harm behaviour and school based mental health interventions. Judi is also interested in how school organisational structures, policies and processes can impact mental health positively or negatively. http://orcid.org/0000-0002$\underline{1054-6758}$ 


\begin{abstract}
Evidence on how different types of social media use contribute to digital stress in early adolescence is lacking. In-depth interviews were conducted with twenty-four 13-14year-olds. Interviews were transcribed verbatim, coded and thematically analysed. Themes were generated based on digital stressors specific to passive social media use (time-wasting and digital guilt, and exposure to harmful content); private social media communication (expectations of availability and unsolicited contact by strangers); and social media broadcasting (expectations of perfection and sexualisation, fear of negative evaluation and risks to privacy). Several digital stressors appeared pervasive in everyday social media use. Bolstering social and emotional resources offline and encouraging effective implementation of privacy settings may improve resilience against several sources of digital stress.
\end{abstract}

Keywords: Social media, Adolescence, Digital stress, Identity, Peer relationships

\title{
Introduction
}

Adolescence is a developmental period typified by intense emotional, cognitive behavioural and social change, which coincides with increased vulnerability to mental illhealth (Blakemore, 2019). The age 13-14 years falls within a period considered early adolescence, characterised by intense pubertal development, egocentrism, a desire for independence and development of sexual identity (Sawyer et al., 2018). Identity development and increased importance of peer relationships and social status (Blakemore, 2019) are two key features of this stage of adolescence which may be particularly salient to social media use (Livingstone, 2008).

Using social media can elicit feelings of digital stress, defined as a mild adverse effect resulting from negative experiences of digital technology use or constant access to social information online (Steel et al., 2020). Digital stress in adolescence has been referred to as an emerging and pervasive source of interpersonal stress for this age group and is longitudinally 
associated with depressive symptoms (Nick et al., 2020). The construct has previously been divided into two types; type 1 stressors are explicitly hostile or malicious uses of social media, such as cyberbullying and public humiliation, and type 2 stressors are aspects of social media use more related to peer socialisation and communication, including feeling pressure to comply with requests for nude photos or sharing passwords, and feeling smothered by excessive online messaging (Weinstein \& Selman, 2016). While cyberbullying involvement is widely recognised as a key mediator in the relationship between social media use and adolescent mental health (Viner et al., 2019), less empirical evidence is available on type 2 stressors. This qualitative study aims to explore experiences of digital stress in the context of narratives of adolescence, with a focus on identity development and peer relationships.

\section{Identity development}

One's identities are "internalized meanings that cluster around how an individual understands themselves, as a social object" relative to another individual (e.g. a close friend), a group of individuals (e.g. one's family or peer group), or a social or demographic collective. These social objects - and therefore one's identities - are situated within cultural and social structures (Mueller et al., 2021, p. 8).

Issues relating to identity development take on particular significance during early adolescence, with an emerging ability to recognise self-perceptions and their consistency or inconsistency with how one is perceived by others (Schwartz \& Petrova, 2018). Healthy identity development is an important public health issue, with "greater consistency and coherence within one's sense of identity... likely to lead to higher well-being, fewer symptoms of anxiety and depression, and lower likelihood of engagement in delinquent and health-compromising behaviours - such as substance misuse, risky sex or violence" (Schwartz \& Petrova, 2018, p. 110). The social world impacts an adolescent's sense of self 
through their identity development, with social inclusion or rejection carrying important implications for mental health and well-being.

Social interaction with others, whether online or offline, is thus a prominent aspect of identity development. According to impression management theory (Goffman, 1959, in Bell, 2019)) we are motivated to make a specific impression on others during social interactions and use our behaviour to manage this in order to be liked by peers, obtain approval and social status and validate a positive self-image. Creating a positive impression involves various selfrepresentative strategies, all of which are facilitated by social media broadcasting or responding to peers' online content. Strategies to create a positive self-representation include conformity to social norms (Fiske \& Taylor, 1991). Social media now plays a key role in shaping these norms and expectations regarding identity expression. Algorithms promote content based on engagement, dictating what is most visible to young users. Social norms are thus widely visible and accessible through viewing social media content posted by peers and others, further influencing how adolescents present themselves in image-sharing (Bell, 2019; Nesi et al., 2021). Positive self-representation is also sought by conveying the most positive image of oneself possible - now facilitated through careful curation of one's own profile and digital enhancement of images - and appreciating or flattering social targets - amplified in frequency and salience through provision of quantifiable feedback on others' content (Bell, 2019; Nesi et al., 2021). With individual personalised profiles, often visible to a global audience, social media in theory provides users with complete control over the strategic presentation of themselves to others. Users can reveal and promote, as well as obscure aspects of their personality and life experiences through the content they publish, in order to shape how they are viewed by others online (Toma, 2013). Users can not only select which pictures of themselves to upload but are able to digitally edit these pictures to enhance their attractiveness or convey a particular mood (Bell, 2019). 
Through specific features such as permanence and quantifiability, social media use is thought to amplify self-presentation related social expectations (Nesi et al., 2018b), representing a source of type 2 digital stress. Self-presentation is fundamental to social media broadcasting, and the efforts and vulnerabilities involved in managing how one is perceived by others are now required more often and to larger and more diverse audiences. Social media broadcasting or direct interactions with others online can be regarded as a form of identity expression and experimentation. With each broadcast, users can have their identity expression either validated or rejected by others - usually peers - in the form of views, likes and comments. Unlike offline interactions, social media provides a permanent record of these validations or rejections, enhancing their salience and enabling users to re-live and ruminate on them (Nesi et al., 2018b). As such, online identity expressions can have substantial implications for the broadcaster's sense of self and well-being (La Sala, 2019).

\section{Peer status and social vulnerability}

As outlined above, social processes play a critical role in adolescent identity development. In addition to mental health risks associated with development of an inconsistent or negative self-concept (Schwartz \& Petrova, 2018), a vulnerability to social exclusion is also thought to contribute to increased susceptibility to mental ill-health in adolescence (Blakemore, 2018).

Experiments from the fields of social psychology and neuroscience have demonstrated a notable increase in risk-taking behaviour during adolescence, particularly in the presence of peers. These findings are thought to relate to a hyper-susceptibility to social evaluation and exclusion during this stage of development (Blakemore, 2018). Salience of social norms among peers is heightened, increasing peer conformity (Andrews et al., 2020). The need to conform is combined with a need to belong (Baumeister \& Leary, 1995), with social status 
among peers taking on particular relevance to one's sense of self and well-being (Oldehinkel et al., 2007).

During adolescence, peer status - in terms of admiration and affection - becomes particularly important in the development of mental health or ill-health, with increased time spent in peer interactions and increased relevance of peers as a basis for social comparisons and for self-appraisal (Oldehinkel et al., 2007). Achievement-related peer status is particularly related to social hierarchies and social comparison. Adolescents typically compare themselves with peers in terms of their intelligence, physical attractiveness, and how much attention they receive from others. Upward social comparisons (in which one perceives one's peers to be superior in a particular domain) are thought to foster feelings of inferiority, in turn leading to low self-esteem and depression (Oldehinkel et al., 2007). Affection-related peer status is more closely related to a need for acceptance and belonging (Baumeister \& Leary, 1995) and a fear of rejection or social exclusion (Blakemore, 2018). Social rejection or ostracization is a key contributor to feelings of loneliness, poor self-esteem and depression (Oldehinkel et al., 2007). An increased focus on peer status in adolescence may increase the risks of upward social comparison and rejection attached to peer interactions. This in turn, may play a significant direct role in the increased vulnerability to mental ill-health during this developmental period, as well as an indirect role through increased participation in risky health behaviours (Blakemore, 2018; 2019; Schwartz \& Petrova, 2018).

Upward social comparison is considered a key process in the relationship between passive social media use and poor adolescent mental health. Increased access to information about others' achievements and status, and comparisons with highly idealised online representations of others, can lead to feelings of envy, rumination and depression (Verduyn et al., 2017). Related to this, increased access to information about other's social activities can foster feelings of loneliness and social exclusion, referred to as a fear of missing out (FoMo; 
Burnell et al., 2019). While peer interactions online may support feelings of social inclusion, quantifiable feedback on content broadcast via social media amplifies awareness and provides an immediate, visible measure of social acceptance or rejection (Bell, 2019; Nesi et al., 2021).

A 'transformation framework' proposed by Nesi et al. (2018a), outlines the unique features of social media that have transformed peer relationships in adolescence. The frequency and intensity of peer interactions have increased in line with the ability to be available online almost constantly, with increased opportunities for digital stress. The qualitative nature of certain experiences has been altered, such as differences in the experience of cybervictimisation as compared to offline victimisation. Social media has also created enhanced opportunities for behaviours that would be unlikely offline, such as the ability to reach and contribute to online communities. Other behaviours are completely novel to the age of social media, including the quantifiable feedback provided in response to broadcast content, or the use of screen-shotting to facilitate "non-consensual distribution of content" (Nesi et al., 2018b, p.299). The framework outlines key transformative features of social media considered less prominent or irrelevant in offline interactions, including: algorithms, publicness, permanence, asynchronicity, availability, absence of cues, visualness and quantifiability (see Nesi et al., 2021).

The importance of peer status, peer influence and peer victimisation in adolescence is thought to be amplified through these various social media features. Examples include the increased opportunity for social comparison based on access to information about peers; amplification of peer victimisation experiences due to the rapid spread, publicness and permanence of cybervictimisation content; and the reinforcement of risky behaviour through quantifiable online reinforcement (Nesi et al., 2018b). The transformation framework thus highlights unique features of social media that shape experiences of peer interactions in 
adolescence, with implications for mental health and well-being (Nesi et al., 2018a; 2018b). In the current qualitative study, we use this framework as a basis for exploring the ways in which different types of social media use have introduced a complexity to the navigation of adolescence more broadly, and the challenges traditionally associated with adolescent identity development and mental health. We apply the transformation framework to qualitative data by way of understanding sources of digital stress specific to different types of social media use in adolescence. We incorporate identity development within the framework in addition to issues relating to peer status and social inclusion or rejection.

\section{Types of social media use and adolescent well-being}

In the wake of conflicting evidence and criticism within the media effects field of a focus on aggregate measures of social media screen-time, much attention has been given to passive social media use and its potential harmful effect on well-being (Burnell et al., 2019; Verduyn et al., 2017). Passive use refers to browsing behaviours - scrolling through news feeds, monitoring the accounts of others and consuming information rather than producing it. Active social media use is considered more 'social' and includes producing and publishing content - also referred to as 'broadcasting' - or communicating directly with others (Kaye, 2021). Evidence from cross-sectional and limited longitudinal and experimental studies suggests that it is passive use of social networking sites specifically that is negatively associated with subjective well-being, most likely by encouraging upward social comparisons, FoMo and feelings of envy (Burnell et al., 2019; Verduyn et al., 2017).

Passive consumption of social media content is particularly conducive to the process of upward social comparisons with others, whereby comparisons are made against those better off than oneself as a form of self-evaluation (Festinger, 1954, in Frison \& Eggermont, 2016). As outlined in the transformation framework (Nesi et al., 2021), unique features of social media amplify these comparisons and potentially exacerbate their harmful effect on well- 
being. Social media users have increased access to information about the social status, activities, achievements and physical attractiveness of others, including peers and family members, but also celebrities and models. With much social media content broadcast by those engaging in strategies to create a positive impression, passive users are often comparing themselves against highly idealised and often digitally enhanced images depicting physical attractiveness and lifestyle. Social media content thus raises the benchmark against which comparisons are made, sometimes to unrealistic or even unattainable standards (Naderer et al., 2021). These social comparisons may be linked to poor self-esteem, depression and lower life satisfaction through rumination and feelings of inferiority, failure, and envy (Mayer et al., 2020; Verduyn et al., 2017).

A recent study (Winstone et al., 2022) developed a four-group typology of adolescent social media users based on their patterns of non-platform-specific private communication, broadcasting and passive browsing. Groups at age 13 included (in order of prevalence) high communicators, moderate communicators, broadcasters, and minimal users. The authors did not identify a group characterised by passive use in the absence of communication-related activities. Testing associations with mental health a year later, broadcasters - the user group characterised by frequent content sharing in addition to very frequent passive use and messaging - had the poorest outcomes, including risk of self-harm, anxiety, depression and poor well-being. Outcomes were best in the two user groups characterised by private communication and passive use but lower levels of broadcasting.

Existing evidence of differential effects of types of social media use on well-being is largely focused on passive use, social comparison and envy. While passive social media use may represent an important source of digital stress, little qualitative research has explored other types of use as sources. In a qualitative focus group study, Bell (2019) explored imagesharing practices among adolescents. Though not explicitly focused on digital stress, findings 
suggested that the desire to achieve a balance between attractive and authentic selfpresentation, and the importance of 'likes' for confidence and social approval, may be sources of stress specific to social media broadcasting.

\section{Objectives of the current study}

Since little is known about early adolescent perspectives of digital stress across different types of social media use, in the current study we analyse qualitative data obtained from interviews with 13-year-olds, covering a range of social media experiences including specific activities as well as screen-time. We approach our reflexive thematic analysis through the theoretical lens of the transformation framework (Nesi et al., 2018a; 2018b), extending this beyond peer interactions to include experiences of identity development in adolescence. In doing so, we aim to enhance understanding of the digital stressors relating to different types of social media use, with implications for transformed experiences of adolescence.

\section{Methods}

Author 1 conducted face-to-face interviews with twenty-four students aged 13-14 years (19 girls and five boys) in February-March 2020. Interviews took place at two English secondary schools in inner-city locations. Recruitment was carried out through teaching staff, with all students in a single year group invited to take part. Students with a range of social media experiences, including non-use, were encouraged to participate. Interviews were conducted with any student who expressed an interest and submitted completed participant and parental consent forms in advance. Three interviews were conducted with individuals; nine were paired interviews and one was with a group of three students. Participants indicated their preferred format in advance and nominated a friend to join them in a paired interview if required. The interviews were audio recorded, took place at school during lesson time and lasted between 45 minutes and an hour. A topic guide (available online at website) was used 
flexibly to ensure consistency in covering a number of core areas for discussion related to social media use and mental health, including different activities, screen-time, night-time use, family and peer dynamics, cyberbullying and body image. Probing included generic encouragement to expand on a statement and suggesting specific social media activities for discussion. Activities included private messaging, group chats, sharing content with different audiences, listening to music, browsing, liking and commenting content, and meeting new people.

Braun and Clarke (2020) advocate six iterative phases to thematic analysis: data familiarisation, data coding, generating initial themes, reviewing and developing themes, refining, defining and naming themes, and writing the report. Immediately after each interview was conducted, author 1 listened to the recording and made notes of initial insights. The interviews were transcribed verbatim and each transcript read thoroughly before being imported into NVivo version 12 for coding. A systematic and inclusive coding process was adopted, with descriptive and interpretative (latent) coding applied flexibly to include unexpected data (Braun \& Clarke, 2020). Following descriptive coding of the first seven transcripts, all codes and initial higher-level categories (clusters of codes) were listed with descriptions and notes. This was shared with author 4 , who coded two transcripts, then provided feedback and additional suggested codes and categories to add further reflexive insight from her own experiences. The remaining transcripts were then coded, with initial codes renamed or combined where appropriate.

Initial themes were constructed using the first few interviews and then reviewed iteratively when applied to the remaining data, with thematic boundaries altered as necessary. Codes and categories clustered about various topics. These shaped initial themes, which were reviewed and discussed with all co-authors. Themes were refined by the selection of illustrative data extracts, checking for coherence and richness of data. Through discussion of 
findings, decisions were taken to drop or combine themes based on how meaningful they were felt to be. In defining themes, movement of codes and categories was accepted to improve coherence. Themes were finalised after further discussion, with interview recordings again revisited to check the applicability and appropriateness of the analysis.

\section{Results}

Three themes were developed through thematic analysis of the qualitative data and are presented in Table 1. Each theme relates to a different type of social media use (passive use, private communication, and broadcasting), encompassing specific sources of digital stress. The themes fit into a narrative of social media transforming longstanding challenges of identity development and social status in adolescence, whereby digital stressors introduced by specific types of social media use are suggested to bring additional consequences for adolescent well-being. Participants interviewed individually did not report notably different experiences of social media use compared to those interviewed in pairs or groups - nor were there school-based differences - so such comparisons are not discussed here. Where illustrative quotes from a single individual are provided, we indicate the participant's gender and interview type.

\section{Table 1}

Types of social media use as different sources of digital stress

\begin{tabular}{lcc}
\hline \multicolumn{1}{c}{ Themes and sub-themes } & $\begin{array}{c}\text { Illustrative } \\
\text { codes }\end{array}$ & Illustrative quotes \\
\hline Digital stress relating to passive & \\
social media use & \\
Time wasting and digital guilt & Time-drain
\end{tabular}


Addictive "I think I spend way too much time...

...it's just addictive, honestly."

(girl, paired)

Exposure to harmful content

Triggering
content

Algorithms

Being

available

Responding

Keeping in

touch

Expectations of availability

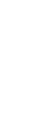

Digital stress relating to private

social media

communication 
Digital stress relating to social

media broadcasting

Expectations of perfection and
Body

sexualisation

dissatisfaction

Presentable

pictures

Judging

others/being

judged

Screen-

shotting

Privacy

concerns
"I feel like that impacts social media because you've got to be so perfect, otherwise you're going to get all, like, criticism from everyone.” (girl, paired)

“...they don’t want to let anything slip up. One thing said can kind of change how people see them." (boy, paired)

Risks to privacy

"If your account is not private on Instagram... it's kind of dangerous, because people can use your photo in lots of different ways." (girl, paired)

\section{Digital stress relating to passive social media use}

\section{Time-wasting and digital guilt}

Participants simultaneously reflected on passive browsing meeting important social and emotional needs, and as a poor use of time, feeling that sometimes no specific needs were met and their time online was futile. Practical uses of social media for entertainment, relaxation, information, feeling inspired and inspiring others, and socialising were perceived by participants to be beneficial to their well-being. Despite an emphasis on reducing screentime as the primary concern for most participants' own use, framing of social media use as 
either a good use of time or "a waste of time" (boy, individual) was dependent on the specific activity or use-context discussed.

Passive use was highlighted as a way of "killing time" (boy, individual) when there is "nothing to do" (girl, paired). This was sometimes presented light-heartedly, with participants laughing about losing track of time or wasting their days. More often, however, this was discussed in the context of wanting to limit or reduce their social media screen-time to focus on other activities they felt to be more useful or rewarding, such as homework, exercise, or face-to-face socialising.

Many participants considered their own passive use to be excessive, though this was largely only considered problematic if it was felt to be meaningless. While many participants expressed a desire to reduce their screen-time, this was caveated if social media activities were felt to be practically useful.

Some participants expressed concerns that social media algorithms contributed to passive use feeling addictive. One participant spoke of feeling trapped by passively scrolling through images on Instagram, conveying a sense of regret at failing to spend her time more wisely. Another described the feeling as "fall[ing] down the rabbit hole" when trying to do homework with YouTube on in the background (girl, paired). Others alluded to tensions between their time spent online and their parents' perceptions of what constitutes a good or healthy use of time, including reading books, playing outside, or meeting new people face-toface. Discussions around struggling to reduce social media screen-time despite feeling they should, reflected a sense of guilt about failure to meet the expectations of parents, teachers, and their own standards of constructive time use.

\section{Exposure to harmful content}

Social media algorithms also contributed to stress associated with viewing harmful content. One participant who alluded to overcoming her own mental health difficulties - 
while finding social media a useful outlet to distract from anxiety - described the additional measures required to ensure her social media use did not trigger problems. This included the need to regularly update settings and preferences to avoid exposure to negative or anxietyprovoking content. The user must mark content as 'inappropriate' to influence platforms' algorithms. Other strategies included unfollowing or blocking certain accounts but were not always effective. This participant described being able to "block" content labelled with specific hashtags that may feel personally triggering. However, blocked content would still appear with a warning that was easily bypassed “...so you're exposed to things that may be hurtful to you" (girl, paired).

The same participant mentioned how certain social media trends, such as threatening 'chain-mail' posts had worsened her existing anxiety as a younger child, requiring her to learn how to effectively navigate social media in order to limit its negative effects. Despite learning techniques for this, and paying heed to disclaimers, there was still a sense of vulnerability to harmful, triggering, or disturbing content if someone failed to tag it appropriately when sharing.

Several other participants mentioned the potential for passive exposure to negative or self-harm related content to perpetuate negative feelings or normalise and encourage selfharm for those already experiencing poor mental health. Some mentioned the potential for a negative cycle in which someone searching for harmful or depressing content is then shown more harmful content as a result of social media algorithms.

...if there's something about depression that you could relate to and you like it, it will just show you more of that, then you wouldn't be able to find any help because, like, on Facebook, it's just showing stuff that is depressing and not good. (boy, paired) Many felt that adolescents who were depressed or self-harming should avoid social media as a result. Two participants discussed a friend who used to watch "suicidal videos" 
and circulate the link to close friends. Despite acknowledging the potential for this to have been a form of help-seeking behaviour, the participants felt that social media worsened their friend's mental health and should have been avoided altogether. This narrative indicated an additional burden placed on adolescents with poor mental health to take responsibility for minimising harm resulting from their social media use. Avoiding harmful social media content may be challenging for those trying to improve their mental health if they are not well-equipped to take on this burden. These users were also felt to be responsible for reporting harmful material to social media companies to protect other young people struggling with their mental health. Exposure to digital stress relating to passive browsing of online content may thus be most acute for those adolescents with poor mental health.

\section{Digital stress relating to private social media communication}

\section{Expectations of availability}

Peer norms regarding expectations of availability and responsiveness featured heavily in discussions around social media messaging. Social media was felt to be a convenient way of keeping in touch with friends and family, and inclusion in group chats sometimes fostered a sense of belonging. However, receiving online messages from peers was a source of stress when young people felt obliged to respond immediately. This stress was compounded when participants were simultaneously participating in multiple online conversations.

The expectation of being available to immediately respond to messages was so normative that participants described having to think of an excuse to leave an online interaction. Not receiving a prompt reply to an online message led some young people to assume a friend was purposely avoiding them. While a broken phone led to an instant feeling of "panic" for one participant - due to the fear that friends would assume she was annoyed or being rude - others described a feeling of relief and relaxation during periods when they 
knew in advance they would be unable to use their phone. Trying to take a break from social media was met with confusion and curiosity from peers.

That's just more stress and it's just less stressful to just stay on it. When you have an excuse like camp where you're like, “Guys, I'm not going to be answering because I'm doing this," it's just so much more relaxing. I enjoy it a lot more than having my phone to be fair. After the first day when you're like, "I wonder what this person is doing or I wonder what latest thing this person has uploaded.” After you stop thinking about that, it's so much nicer. (girl, group)

\section{Unsolicited contact by strangers}

Participants broached experiences and perceived risks of victimisation by strangers. Whereas children have long been warned not to talk to strangers, the global reach of social media provides overwhelming opportunities for young users to receive unsolicited contact from strangers, sometimes including regular sexual harassment. With Snapchat including a function allowing location tracking, adolescents must also now consider who might be able to see where they are while using social media. Participants mentioned their own - as well as their parents' and teachers' - fears of strangers using this information to track them down to cause harm.

Vulnerability to abuse via social media was discussed both hypothetically and in terms of participants' own experiences.

You know the other day yes, this person sent me, "Hey." With two kisses. He was about 46. I ignored him yes, and I was like, “Okay, bye.” Then the next day I get another Snap. I'm like, “Ah, no thank you.” (girl, paired)

Participants described receiving unsolicited sexualised contact (such as nude pictures) by strangers as “disgusting”, “disturbing”, "rank", "weird” and "dodgy”. Although some insisted "it's not a big thing at all", participants sometimes censored their experiences from 
adults at home or at school in case they were stopped from using social media. Despite claims that this regular sexual harassment did not particularly bother them, their use of emotive language and acknowledgement of the regularity with which this occurs indicated otherwise. Participants clearly understood the seriousness of this harassment through the implicit recognition that it could lead parents or teachers to take drastic measures to intervene.

Whether discussing sexual harassment or more seemingly innocuous (though still “odd", "creepy", or "uncomfortable") unsolicited contact from strangers, participants seemed extremely aware of the need to be careful when using social media. In most cases, these feelings led to young people making changes to privacy settings, blocking and reporting users to social media companies, or simply ignoring requests. For other participants, the risks discussed were motivation for limiting or not using social media at all. Whether from direct experience, or a narrative perpetuated by parents and teachers, participants expressed a constant sense of vulnerability to harm. These aspects of vulnerability related particularly to a sense of stranger danger from being contactable online, which, while typically low level, seemed ever present at the forefront of participants' minds.

\section{Digital stress relating to social media broadcasting}

\section{Expectations of perfection and sexualisation}

Young people were felt to experience pressure for public self-disclosure in terms of sharing 'selfies', with an emphasis for girls on perfectionism and presenting themselves in ways that conform to expectations of beauty and sexuality. Pressure to conform seemed to be accentuated by exposure to such expectations through viewing social media content. Participants' comments divulged a clear sense of vulnerability to threats related to their selfpresentation when broadcasting on social media, with a general fear of being judged for their appearance or comments they made on peers' content. 
Social media affords opportunities but also pressure for adolescents to "post more of themselves" (girl, paired) for an audience of peers or beyond. Online broadcasting offers young people control over editorial decisions to present themselves as they wish to be seen by others. Quantifiable likes and views may contribute to the broadcaster's self-esteem when internalised ("I guess it's like I care more about what other people think of me than what I think of myself"' (girl, paired)). Furthermore, the visibility of these likes and views can represent a status symbol or beacon of popularity ("the more likes you get, the more people like you" (boy, individual)). As such, issues around self-presentation appeared heightened in frequency, intensity and importance for adolescents in the digital age.

Impression management strategies employed by participants included avoidance of broadcasting to save face and avoid judgement, use of filters, curation of profiles with deletion of undesirable or unpopular content, and selection of desirable images to share.

Presentable pictures. Yes, because obviously you don't want, when you post you want people to think that obviously you portray yourself well and you think of yourself well and you look after yourself. Obviously that all depends on the photo you choose to post... (girl, paired)

As well as facilitating impression management, social media use may also present challenges for those young people who have not yet developed expertise in audience management and may cross over into excessive self-inflation. Others' perceived excessive self-presentation or concerns regarding popularity - such as using strategies to gain followers who are not actually friends - were sometimes pitied as a symptom of low self-esteem or frowned upon as unhealthy and "fake". The common practice of editing and enhancing selfies using filters was perceived negatively by some for its inauthenticity in selfrepresentation. 
Despite the assumed benefits of young people having control over their online selfpresentation, some participants spoke of the pressure to modify this to be perceived favourably, with selfies receiving more likes than landscapes. Previous generations have encountered gender stereotyping and pressure for girls to present themselves in ways that adhere to "the expectation of beauty" (girl, paired) - being "pretty", "skinny", or "perfect" as well as in sexualised contexts. Describing a peer's age-inappropriate use of TikTok, one participant said, "let's just say she was like 10 when she started doing some dances for some boys...... There is this boy dancing and she is licking up her lips and I'm like, "How old are you again?"'. Another participant noted that algorithms promoted content presenting girls or women wearing tight-fitting clothes, creating pressure to self-objectify in order to improve one's social status online.

\section{Fear of negative evaluation}

A sense of vulnerability emerged from participants' narratives around social media broadcasting, focusing on external evaluations of content. Participants on numerous occasions voiced concerns around their own and others' vulnerability to being "judged" or "mocked" by both peers and complete strangers.

For some, this was a necessary part of posting social media content and reflected wider societal norms for perfectionism and social evaluation. For others, this fear of judgement was given explicitly as a reason for avoiding social media broadcasting and preferring to use social media passively or for direct communication only.

...some people might have anxiety and they might feel scared to post, though, because they feel like they're going to get hate from other people and they feel like other people are going to judge them, so they feel more insecure. (girl, paired) Fear of negative evaluation led other participants to adapt their social media use by sharing content privately to trusted friends rather than to a wider network. Use of privacy 
settings, including broadcasting 'private stories' to close friends on Instagram seemed to provide a safe space in which young people could appear unfiltered without fear of embarrassment or negative evaluation “...because I know they wouldn’t [judge me]” (girl, paired). As such, these private sections of an adolescent's social media presence may be an important contribution to enabling identity experimentation and development within a less judgemental circle of close friends.

Fear of judgement seemed to relate particularly to physical appearance and to some extent a fear of body-shaming or being bullied, both from personal experience and hypothetically based on observing peers' experiences. Participants also expressed a fear of being judged on text-based social media broadcasts, such as a comment being (mis)interpreted as bullying or "slipping up" by saying the wrong thing or expressing a controversial opinion. Again, this fear was either given as a motivation for avoiding social media broadcasting or as an exacerbating factor in peer conflict.

Peers who broadcast their mental health difficulties on social media were considered particularly vulnerable to being negatively judged based on their posts, with behaviour often framed as "attention-seeking". Describing a peer broadcasting herself crying on TikTok, one participant noted "it's like she's trying to make everything all about her". This was echoed in others' comments describing an older girl broadcasting self-harm-related quotes and videos of her own self-harm. Rather than eliciting peer support or access to more formal services, this broadcasting seemed more often to backfire through peers' dismissal of the behaviour as attention-seeking, negative reactions to the content, or increased vulnerability to bullying. In some cases, the peer experiencing difficulties was ostracised from friendship groups when offers of concern or support resulting from an online post were rejected.

A narrative of disdain relating to those broadcasting difficulties online was taken further by some participants who considered the potential for this behaviour to harm others 
through normalising self-harm or "spread[ing] that kind of behaviour to other people" (girl, paired).

Evaluation-related concern underpinned discussions, including both the desire for their posts to receive likes or views, and anxiety about how peers might perceive them based on how they look in posts or comments they make online. As such, the constant underlying fear of appearance- and status-related evaluation associated with social media broadcasting may be a key source of digital stress in early adolescence, with specific challenges faced by those using broadcasting as a form of help-seeking behaviour.

\section{Risks to privacy}

Almost all participants spontaneously raised the issue of privacy concerns relating to social media broadcasting. For some participants, they were reassured of protection from potential harms of social media by setting their accounts to 'private'. The multiple negatively framed mentions varied in severity, from the desire to keep posts restricted to trusted close friends; feelings of regret and shame attached to mistakenly publishing personal details in a public Instagram post; awareness of the possibility of having accounts hacked, selfies altered and misused, or private sensitive messages screen-shotted and shared maliciously; being harassed by strangers asking for or sending sexually explicit pictures in a direct message; and a mindfulness that strangers might use social media information to track you down and cause you harm.

'Screen-shotting' and re-distribution of content without permission, while unique to those experiencing adolescence in the digital age, was viewed by some as analogous to offline gossip. One participant described this as "like when you tell someone not to tell someone, and they go and tell someone" (girl, paired). Screen-shotting was seen as a key component in fostering mistrust, complicating or exacerbating peer conflict (see also authors, 2021). For some participants, the unease attached to screen-shotting was a reason to avoid 
social media broadcasting or online messaging, preferring to communicate with friends in person, which was felt to be "more private". Using screen-shotting to spread gossip was felt to underly the perception that social media "could make or break relationships" (boy, paired).

This cohort of adolescents must navigate relatively new and complex issues regarding data privacy (which almost all participants volunteered as a potential harm of social media), ever evolving functionality of apps, and long-term implications of publishing their own content. As one participant noted, “you don't really know what people can do with your pictures nowadays. So, like, you should be quite careful on social media” (girl, paired). Broadcasting - posting selfies in particular - was described as "dangerous" due to the risks of publishing private information such as addresses. Adolescents also now need to factor in the permanence of visibility of any potential mistakes, with some participants preferring to avoid sharing photos and videos via social media, commenting, or contributing to online discussions for fear of being misinterpreted, or repercussions that might damage their selfimage or future career.

\section{Discussion}

\section{Key findings}

Adolescent narratives presented here referred to broad experiences of early adolescence in the age of social media and incorporated several examples of digital stress. Different types of social media reflected different sources of stress. Passive browsing of online content represented a perceived time-wasting activity in many cases, accompanied by a sense of guilt. Passive social media use was also conveyed as burdensome for adolescents experiencing poor mental health, who had specific challenges in navigating algorithms to avoid exposure to harmful content. The use of social media to communicate directly and privately with others is beneficial for social connectedness. However, this type of use was also a source of digital stress relating to peer expectations of availability and to the receipt of unsolicited - often 
sexual - contact from strangers. Social media broadcasting - sharing text- or image-based content online - was a source of stress relating to concerns around expectations of perfection and sexualisation, anticipation or fear of negative evaluation, and risks to privacy.

Findings relating to fear of judgement and expectations of availability are consistent with existing literature on digital stress (Steele et al., 2020). Unlike literature reviewed by Steele et al. (2020), however, we did not find strong evidence of stress resulting from fear of missing out or negative social comparison. While fear of missing out was not explicitly explored in these interviews, our findings indicate that resilience to negative social comparison may increase with age, experience, or digital literacy. Adolescents in this sample appeared acutely aware of the psychological risks associated with comparing themselves to unrealistic online imagery.

\section{Differential transformation of adolescence: implications for well-being}

Several participants in our study were keen to record their view that the potential harm of social media is overestimated by older generations who did not understand the online world. Reflections on participants' own use emphasised the practical and beneficial nature of social media as a channel for providing entertainment, a way to pass the time and relax, be inspired, stay updated with current affairs of particular interest, to keep a record of day-to-day events and good times and to keep in touch with friends and family. Both asynchronous texting and synchronous communication via telephone calls have been to a large extent usurped by similar direct messaging functions on Instagram, Snapchat and WhatsApp to communicate privately with others. The functions of hand-written diaries and photograph albums are now similarly met by personal feeds and timelines to record events and feelings, through similar features of permanence and visualness. Indeed, others have highlighted the additional benefits of availability of information on social media, in terms of facilitating retrieval of information (Nesi et al., 2021). Passive browsing of carefully curated and edited content in 
magazines has been replaced by scrolling passively through content posted by peers and celebrities. Similarly, many of the problems popularly associated with social media already existed in offline experiences of adolescence, with family arguments or rules about social media screen-time replacing those related to time spent in front of television screens or computer games (Orben, 2020a); online bullying often an extension of offline bullying (Wolke, 2017); and the sometimes-fraught dynamics of friendship groups playing out online rather than or in addition to face-to-face (Nesi et al., 2021).

However, social media has introduced additional complexities to the daily concerns of today's adolescent. Existing concerns appear to be exacerbated through several unique features of social media. In their transformation framework, Nesi et al. (2021) list these as: publicness, permanence, asynchronicity, availability, absence of cues, visualness, quantifiability and algorithms (this last feature being an update to the framework originally proposed (Nesi et al., 2018a, 2018b)). Our findings provide detailed support for the transformation framework (Nesi et al., 2018a), whereby social media has altered the experience of peer interactions in adolescence. We extend this framework by examining the unique social media features relevant to specific types of use that have differentially transformed experiences of adolescence more broadly, relating to digital stressors associated with identity development and vulnerability to social exclusion. Our findings indicate that different features are likely to be of particular relevance to different social media user types (Winstone et al., 2022). In addition to algorithmic issues regarding cumulative exposure to harmful content, algorithms influence social norms that dictate production and broadcasting of social media content by promoting content based on its popularity. Algorithms thus both shape the experiences of those using social media passively (in terms of exposure to specific content) but likely shape the content broadcasters share, for example through increasing pressure for attractive and sexualised self-representations. Quantifiability - numbers of 
views, followers, likes and comments - provide a tangible and visible measure of social status (popularity) to broadcasters as well as being a metric of acceptance or rejection of broadcasters' identity expressions and experiments (Bell, 2019). Concerns around representations of one's physical appearance and social status - amplified online by publicness, permanence and visualness - have mental health implications for adolescent social media broadcasters. Features such as permanence and visualness enable passive social media users to anonymously observe high status peers, increasing opportunities for upward social comparison (Nesi et al., 2021).

Based on our findings, we suggest that replicability and reach are additional key features of social media that warrant inclusion in the transformation framework when applied more broadly to experiences of adolescence beyond peer interactions. Replicability relates specifically to the ability of others to 'screen-shot' messages and social media content, distribute this widely (potentially without consent or knowledge of the broadcaster) and contribute to gossip, peer conflict or data privacy breaches. Although Nesi et al. (2021) include consideration of screen-shots within the feature of permanence and availability, narratives relating to this feature of social media use (in terms of content sent in private messages or broadcast to larger groups of followers) were so prominent that we consider it a distinct transformative feature. Key to impression management - whether in online or faceto-face interactions - and an important social media affordance, is the concept of information control. Social media features enable young people to present themselves in accordance with a positive self-image through editorial decisions regarding online broadcasting, control of information and pace of asynchronous communications or saving face through avoidance of broadcasting (Feaster, 2010). However, the advent of screen-shotting undermines privacy information control, whereby any audience member is now able to reproduce and edit the original content. Screen-shotting thus represents a threat to young people's ability to control 
their self-image, and an important source of digital stress for those wishing to share content online.

Reach is linked to the features of availability and publicness discussed by Nesi et al. (2021), but again played such a prominent role in narratives of vulnerability to harm that we feel it warrants inclusion in its own right. Reach can refer to a positive online accessibility to otherwise remote communities (for example, providing unique opportunity for a young adolescent in England to interact with global strangers of all ages who share similar interests). However, reach also refers to the ability for adolescent social media users to receive unsolicited contact from strangers, contributing to feelings of vulnerability to predatory behaviour.

Such features and associated concerns have altered the experience of adolescence in the age of social media. Replicability is in some ways analogous to a more intense and public form of offline gossip. Reach both enhances opportunities for identity development through participation in special interest communities, and increases anxieties attached to stranger danger. Combined, the transformative effects described above lend a more demanding cognitive and emotional complexity to the navigation of early adolescence compared with previous generations in their offline experience of this important stage of life. Such pressures may represent a "continuous, subtle strain on the identity of the younger generation" (Mayer et al., 2020, p.9).

\section{Type 1 and type 2 digital stressors}

In this sample of young adolescents who generally did not report involvement in cyberbullying, we found limited evidence of type 1 digital stressors. Those type 1 stressors experienced related to online sexual harassment. We found stronger evidence of a wide range of chronic stressors relating to everyday social media practices. These are more aligned with type 2 stressors as described by Weinstein and Selman (2016). Our findings include some 
evidence that particular individuals and groups may be more vulnerable to negative effects of digital stress. Adolescents experiencing poor mental health or with a history of self-harm may be faced with additional challenges of safely navigating social media use, both in terms of peer reception of help-seeking broadcasts, and avoidance of harmful content when passively browsing online. Those prone to excessive reassurance seeking - who may use social media particularly intensively for broadcasting and communication - may also be at increased risk of social exclusion through being perceived as irritating or smothering peers.

In addition to these risk factors for vulnerability to digital stress, we found evidence of more chronic exposure to stress at the population level due to normative pressures accentuated by the social media context. These include guilt related to a discourse of screens as an inherently bad use of time, unrealistic expectations of availability for peer communication, anticipation of harm, pressure to self-publicise, pressure to conform to expectations of perfection and sexualisation, expectations of evaluation and fear of it being negative, and the pressure to avoid risks to privacy. While these persistent digital stressors may be pervasive in young users engaging in different types of social media use, our findings also indicate measures that may reduce exposure to stress or improve resilience where exposure is unavoidable.

\section{Limitations and future research directions}

Our findings should be interpreted in light of the following limitations. First, boys were underrepresented in our sample, making it unfeasible to explore in depth potential gender differences in experiences of digital stress. However, a broad range of views and experiences were captured in the sample, which were sufficient to explore stressors as mechanisms underpinning the relationship between different types of social media use and poor adolescent well-being. Whilst the sample of adolescents in this study included a broad range of experiences, further qualitative research to enhance understanding of different types of 
social media use might benefit from a more direct investigation of specific user types using purposive sampling. Our sample included young people from a single year group (age 13-14years). Experiences of, and vulnerability or resilience to digital stress may vary across adolescence and caution should be applied in generalising findings to other age groups.

Our findings highlight the potential importance of privacy settings in mitigating some of the harms associated with social media use, not only in terms of avoiding data misappropriation or unsolicited contact from strangers but to reduce evaluation-related anxiety. Empirical evidence is required to understand the motivations and implications of broadcasting to different audiences - close friends (in one-to-one or group messages, or as a defined follower group), family members, wider peers, online communities, or the general public.

In adolescent narratives around their experiences of using social media, screen-shotting, cyber-sexual harassment and fear of judgment emerged as key factors that may have negative implications for well-being. Further research is required to explore the role of screen-shotting in cyberbullying for example, to test its prevalence, and the psychosocial impact of having content replicated and re-distributed without consent.

\section{Implications for digital literacy and resilience}

Our findings indicate several key messages for young adolescents about minimising digital stress and supporting resilience in negotiating stressors, with digital literacy and sex and relationships curricula useful vehicles for schools-based delivery. We recommend a focus on i) understanding the role of algorithms in shaping social norms, promoting specific content and feelings of time drain; ii) awareness of others' self-presentation strategies online and mindfulness of the appropriateness of content as a source for social comparison; iii) importance of effective management of privacy settings to avoid exposure to unsolicited contact and mitigate the risk of negative evaluation, social exclusion and data 
misappropriation; iv) issues around consent with regards data misappropriation and unsolicited sexual contact; v) mindfulness of excessive reassurance-seeking (and its social risks) and over-connectedness in disproportionate levels of communication or broadcasting; vi) encouragement and guidance around removal of unnecessary social media features, including visibility of other people's views, likes, followers or comments, or the 'read receipt' function to show when someone has viewed a message; vii) appreciation of the opportunities and value of social media use as well as its risks, with a focus on the importance of healthy and meaningful social relationships offline to benefit most from social media. We further suggest that adolescents with existing mental health concerns may need specific support to safely navigate social media use and manage digital stress.

The importance of good offline social resources in mitigating digital stress has been discussed elsewhere (authors, 2021). This includes the importance of parents and teachers taking care in discussing social media use for its positive opportunities rather than dismissing all use as a waste of time. Related to this is the need for young people to feel comfortable approaching trusted adults for advice when encountering online harms without fear of repercussion. Social media is often framed in public discourse as treacherous and harmful (O'Reilly et al., 2018). Much of the reported digital literacy education received from school broadly consistent with the boundaries set by many parents of the young people in our study - appears to be focused on risk. In addition to a contemporary form of stranger danger, participants were fearful of data misappropriation. With social media use now almost ubiquitous among young people, adolescents may currently be undergoing an important transitional phase of development in a complex social environment tainted by a sense of fear and vulnerability. It may be beneficial to re-frame the social media discourse to highlight the potential for positive experiences when privacy settings are appropriately applied, enabling young people to communicate and share uncontrived content that expresses and develops 
their identity in a safe space, with good friends. Passive social media use might be freed from its overwhelmingly negative label, highlighting the potential benefits of using social media for entertainment, inspiration or relaxation in addition to strategies for avoiding or coping with harmful content. A more important emphasis for digital literacy might be around improving offline social resources and the implementation of privacy settings - and simultaneous consideration of audience - in order to maximise the benefits of social media use, such as self-disclosure and less risky identity experimentation.

\section{References}

Andrews, J. L., Foulkes, L., \& Blakemore, S.-J. (2020). Peer influence in adolescence: Public-health implications for COVID-19. Trends in Cognitive Sciences, 24(8), 585587. https://doi.org/10.1016/j.tics.2020.05.001

Baumeister, R. F., \& Leary, M. R. (1995). The need to belong - desire for interpersonal attachments as a fundamental human-motivation. Psychological Bulletin, 117(3), 497529. https://doi.org/10.1037/0033-2909.117.3.497

Bell, B. T. (2019). "You take fifty photos, delete forty nine and use one": A qualitative study of adolescent image-sharing practices on social media. International Journal of ChildComputer Interaction, 64-71. https://doi.org/10.1016/j.ijcci.2019.03.002

Blakemore, S. (2018). Avoiding social risk in adolescence. Current Directions in Psychological Science, 27(2), pp. https://doi.org/10.1177/0963721417738144

Blakemore, S. J. (2019). Adolescence and mental health. Lancet, 393(10185), 2030-2031. https://doi.org/10.1016/S0140-6736(19)31013-X

Braun, V., \& Clarke, V. (2020). One size fits all? What counts as quality practice in (reflexive) thematic analysis? Qualitative Research in Psychology. https://doi.org/10.1080/14780887.2020.1769238 
Burnell, K., George, M. J., Vollet, J. W., Ehrenreich, S. E., \& Underwood, M. K. (2019). Passive Social Networking Site Use and Well-Being: The Mediating Roles of Social Comparison and the Fear of Missing Out. Cyberpsychology-Journal of Psychosocial Research on Cyberspace, 13(3). https://doi.org/10.5817/cp2019-3-5

Feaster, J. C. (2010). Expanding the Impression Management Model of Communication Channels: An Information Control Scale. Journal of Computer-Mediated Communication, 16(1), 115-138. https://doi.org/10.1111/j.1083-6101.2010.01535.x

Fiske, S. T., \& Taylor, S. E. (1991). Social cognition, 2nd ed. New York, NY, England: Mcgraw-Hill Book Company.

Frison, E., \& Eggermont, S. (2016). "Harder, Better, Faster, Stronger": Negative Comparison on Facebook and Adolescents' Life Satisfaction Are Reciprocally Related. Cyberpsychology Behavior and Social Networking, 19(3), 158-164. https://doi.org/10.1089/cyber.2015.0296

Kaye, L. K. (2021). Exploring the "socialness" of social media. Computers in Human Behavior Reports, 3(100083). https://doi.org/10.1016/j.chbr.2021.100083

La Sala, L. (2019). Developing a Framework for Understanding Adolescent Behaviour on Social Media: A Sequential Exploratory Mixed Methods Study. (PhD), Swinburne University of Technology, Australia.

Livingstone, S. (2008). Taking risky opportunities in youthful content creation: Teenagers' use of social networking sites for intimacy, privacy and self-expression. New Media \& Society, 10(3), pp. https://doi.org/10.1177/1461444808089415

Mayer, G., Alvarez, S., Gronewold, N., \& Schultz, J.-H. (2020). Expressions of individualization on the internet and social media: Multigenerational focus group study. Journal of Medical Internet Research, 22(11), ArtID e20528. https://doi.org/10.2196/20528 
Moreno, M. A., \& Uhls, Y. T. (2019). Applying an affordances approach and a developmental lens to approach adolescent social media use. Digital Health, 5. https://doi.org/10.1177/2055207619826678

Mueller, A. S., Abrutyn, S., Pescosolido, B., \& Diefendorf, S. (2021). The Social Roots of Suicide: Theorizing How the External Social World Matters to Suicide and Suicide Prevention. Front Psychol, 12, 621569. https://doi.org/10.3389/fpsyg.2021.621569

Naderer, B., Peter, C., \& Karsay, K. (2021). This picture does not portray reality: developing and testing a disclaimer for digitally enhanced pictures on social media appropriate for Austrian tweens and teens. Journal of Children and Media. https://doi.org/10.1080/17482798.2021.1938619

Nesi, J., Choukas-Bradley, S., \& Prinstein, M. J. (2018a). Transformation of Adolescent Peer Relations in the Social Media Context: Part 1-A Theoretical Framework and Application to Dyadic Peer Relationships. Clinical Child and Family Psychology Review, 21(3), 267-294. https://doi.org/10.1007/s10567-018-0261-x

Nesi, J., Choukas-Bradley, S., \& Prinstein, M. J. (2018b). Transformation of Adolescent Peer Relations in the Social Media Context: Part 2-Application to Peer Group Processes and Future Directions for Research. Clinical Child and Family Psychology Review, 21(3), 295-319. https://doi.org/10.1007/s10567-018-0262-9

Nesi, J., Dredge, R., Maheux, A. J., Roberts, S. R., Fox, K. A., \& Choukas-Bradley, S. (2021). Peer Experiences via Social Media. Reference Module in Biomedical Sciences. https://doi.org/10.1016/B978-0-12-818872-9.00046-7

Nick, E. A., Kilic, Z., Nesi, J., Telzer, E. H., Lindquist, K. A., Prinstein, M. J., (2021). Adolescent digital stress: Frequencies, correlates, and longitudinal association with depressive symptoms. Journal of Adolescent Health. https://doi.org/10.1016/j.jadohealth.2021.08.025 
Oldehinkel, A. J., Rosmalen, J. G. M., Veenstra, R., Dijkstra, J. K., \& Ormel, J. (2007). Being admired or being liked: Classroom social status and depressive problems in early adolescent girls and boys. Journal of Abnormal Child Psychology, 35(3), 417427. https://doi:10.1007/s10802-007-9100-0

Orben, A. (2020). The Sisyphean Cycle of Technology Panics. Perspect Psychol Sci, 15(5), 1143-1157. https://doi:10.1177/1745691620919372

O'Reilly, M., Dogra, N., Whiteman, N., Hughes, J., Eruyar, S., \& Reilly, P. (2018). Is social media bad for mental health and wellbeing? Exploring the perspectives of adolescents. Clinical Child Psychology and Psychiatry, 23(4), 601-613. https://doi:10.1177/1359104518775154

Sawyer, S. M., Afifi, R. A., Bearinger, L. H., Blakemore, S. J., Dick, B., Ezeh, A. C., \& Patton, G. C. (2012). Adolescence: a foundation for future health. Lancet, 379(9826), 1630-1640. https://doi:10.1016/S0140-6736(12)60072-5

Schwartz, S. J., \& Petrova, M. (2018). Fostering healthy identity development in adolescence. Nature Human Behaviour, 2(2), 110-111. https://doi:10.1038/s41562-

\section{$\underline{017-0283-2}$}

Steele, R. G., Hall, J. A., \& Christofferson, J. L. (2020). Conceptualizing digital stress in adolescents and young adults: Toward the development of an empirically based model. Clinical Child and Family Psychology Review, 23(1), 15-26.

\section{https://doi:10.1007/s10567-019-00300-5}

Toma, C. (2013). Psychological benefits and costs: A self-affirmation framework for understanding the effects of Facebook self-presentation. In C. Cunningham (Ed.), Social networking and impression management : self-presentation in the digital age (pp. 227-246). Lanham: Lexington Books. 
Valkenburg, P. M., \& Oliver, M. B. (2019). Media effects theories: An overview. In M. B. Oliver, A. A. Raney, \& J. Bryant (Eds.), Media Effects: Advances in Theory and Research: Fourth Edition (4th ed., pp. 16-35). New York: Routledge.

Verduyn, P., Ybarra, O., Resibois, M., Jonides, J., \& Kross, E. (2017). Do Social Network Sites Enhance or Undermine Subjective Well-Being? A Critical Review. Social Issues and Policy Review, 11(1), 274-302. https://doi:10.1111/sipr.12033

Viner, R. M., Aswathikutty-Gireesh, A., Stiglic, N. z., Hudson, L. D., Goddings, A., Ward, J. L., \& Nicholls, D. E. (2019). Roles of cyberbullying, sleep, and physical activity in mediating the effects of social media use on mental health and wellbeing among young people in England: a secondary analysis of longitudinal data. The Lancet Child \& Adolescent Health. https://doi:10.1016/S2352-4642(19)30186-5

Weinstein, E. C., \& Selman, R. L. (2016). Digital stress: Adolescents' personal accounts. new media \& society, 18(3), 391-409. https://doi.org/10.1177/1461444814543989

Winstone, L., Mars, B., Haworth, C. M. A., Heron, J., \& Kidger, J. (2022). Adolescent social media user types and their mental health and well-being: Results from a longitudinal survey of 13-14-year-olds in the United Kingdom. JCPP Advances, e12071. https://doi.org/10.1002/jcv2.12071

Winstone, L., Mars, B., Haworth, C., \& Kidger, J. (2021). Social media use and social connectedness among adolescents in the United Kingdom: a qualitative exploration of displacement and stimulation. BMC public health, 21(1),

1736. https://doi.org/10.1186/s12889-021-11802-9

Wolke, D. (2017). Cyberbullying: how big a deal is it? Lancet Child \& Adolescent Health, 1(1), 2-3. https://doi:10.1016/S2352-4642(17)30020-2 